\title{
2 Years into the Pandemic: What Did We Learn About the COVID-19 and Cerebellum?
}

\author{
Aasef G. Shaikh ${ }^{1,2} \cdot$ Mario Manto ${ }^{3,4} \cdot$ Hiroshi Mitoma ${ }^{5}$ \\ Published online: 28 January 2022 \\ This is a U.S. government work and not under copyright protection in the U.S.; foreign copyright protection may apply 2021
}

\begin{abstract}
Highly contagious pandemic due to novel coronavirus SARS-CoV-2, COVID-19 has significantly affected humankind. At the onset of the pandemic, it was believed that it primarily affects the respiratory and hematological system, and has minimal influence on the human brain, even less so on the cerebellum. It was thought that the effects of a pandemic on cerebellar disorders would be the same as it would affect any other chronic neurological disease. It turned out that our understanding of the effects of COVID-19 on the cerebellar system was premature. Over the last 2 years, we appreciated many diverse and direct effects of COVID-19 on cerebellar function. SARS-CoV-2 affects the cerebellum via direct viral invasion, but even more so through its effects on immune, hematological, and metabolic pathways. Increasing evidence suggested the indirect effects of COVID-19 on preexisting chronic cerebellar disease due to lack of in-person care and social isolation. This editorial concisely summarizes critical literature on COVID-19 and the cerebellum published over the last 2 years.
\end{abstract}

The zoonotic corona virus called severe acute respiratory syndrome coronavirus 2 (SARS-CoV-2) caused a pandemic coronavirus disease 2019 (COVID-19). The pandemic initially presented with severe pulmonary dysfunction, acute respiratory collapse, and multiple systems failures; about $36 \%$ of COVID-19 patients were reported to have central or peripheral nervous symptoms and sometimes non-specific deficits such as impairment of smell and taste, headache, fatigue, and dizziness $[1,2]$. Reported neurological deficits were directly related to COVID-19 infection, changing the body reaction to the virus, and immune-mediated hyperactivity. On the other hand, those with chronic neurological conditions, including the cerebellar ataxias, were

Aasef G. Shaikh

axs848@case.edu

1 Department of Neurology, University Hospitals, Case Western University, 11100 Euclid Avenue, Cleveland, OH 44022, USA

2 Louis Stokes Cleveland VA Medical Center, Cleveland, $\mathrm{OH}$, USA

3 Unité Des Ataxies Cérébelleuses, Service de Neurologie, CHU-Charleroi, 6000 Charleroi, Belgium

4 Service Des Neurosciences, University of Mons, 7000 Mons, Belgium

5 Department of Medical Education, Tokyo Medical University, Tokyo, Japan indirectly affected due to scarcity of in-person patient care, social isolation, and lack of in-person rehabilitation [3, 4]. The latter resulted in many new means of providing remote care, teleneurology, and telerehabilitation synchronously while the provider is available for the live session or prerecorded asynchronous video visits [3-5]. Initially considered a respiratory and hematological disease, COVID-19 soon declared its impact on the nervous system. The acute effects of COVID-19 on the central nervous system included ischemic and hemorrhagic cerebral disease, encephalopathies, and seizures [6]. At the onset of the disease, the role of SARS-CoV-2 was still thought to be minimal in the pathogenesis of the cerebellar disease, and it was believed that it does not commonly cause ataxias but it would impact the cerebellar ataxias just in a way it would affect any chronic neurological disorder $[3,4,7]$. The perspective about the effects of COVID-19 on cerebellar function changed over the last 2 years. Here, we will summarize such progress, particularly focusing on how SARS-CoV-2 affects the cerebellar system, the direct and indirect effects of COVID-19 on cerebellar physiology, and how the disease has affected the cerebellar preexisting cerebellar states.

The pathophysiology of COVID-19 is relatively less understood; the mechanistic clarity is even more deficient in the central nervous system. Studies of post-mortem examinations are complex due to laboratory safety concerns. SARS$\mathrm{CoV}-2$ is frequently detected in the cerebrospinal fluid (CSF) 
and brain parenchyma, suggesting its neuroinvasive potential [8]. One study found that the viral invasion is robust in the olfactory system and the brainstem, but less so in the cerebral cortex and the cerebellum [8]. On the contrary, a different study revealed increased viral invasion into the parietal cortex and the cerebellum [9]. A post-mortem study examining the brain tissue from those who had acute SARSCoV-2 infection revealed territorial infarcts in $14 \%$, while majority had astrogliosis [10]. The activation of microglia and infiltration by cytotoxic T-lymphocytes was prominent in the brainstem and cerebellum. Regions of brainstem cranial nerve nuclei had presence of SARS-CoV-2 protein [10].

Increased parenchymal viral infiltration is correlated with severe microgliosis and lymphocytic infiltrations. Besides the direct effects of viral penetration, COVID-19 affects the central nervous systems in many other ways. A wide range of clinical neurological deficits in the acute, as well as chronic phase of COVID-19, is accounted for by large variability in the neuropathological lesions. There are major vascular changes in the cerebral cortex, while there is also substantial meningeal and perivascular inflammation of the cortical parenchyma with hypoxic cellular injury. The cerebellum and brainstem often show perivascular lymphocytic inflammation of CD8-positive T cells mixed with CD68-positive macrophages [11]. The inflammatory changes typically target disrupted vascular walls suggesting prominent role of microvascular injury in COVID-19-induced neurological comorbidity [11]. The hypoxic changes are noticeable in COVID-19 patients with brainstem and cerebellar involvement [12].

The neurological events found on MRI of COVID-19 patients can be diffuse and be present through the cerebral cortex, brainstem, and cerebellum. The classic MRI findings in COVID-19 patients include isolated oval-shaped lesions in the corpus callosum, bilateral basal ganglia, hemorrhage, ischemic lesions including that of the cerebellum, and vasogenic edema extending to the cerebral peduncles, pons, and ventricles [13]. The cerebellar pathological changes can sometimes be initial deficits, although they may remain subclinical and do not manifest at the time of presentation. For example, the diffusion model evaluated the spatial distribution of the events depicting a strong association between predicted and actual events. The events were prominently seen in the bilateral superior temporal cortex and precentral and lateral occipital cortices. The events were significantly associated with diffusion from the bilateral cerebellum in addition to the putamen. The study shows that transconnectom diffusion of pathology occurs via structural network connections of the cerebellum [14]. The vascular microhemorrhages are not uncommon etiology of cerebrovascular pathology in those with COVID-19. Several etiologies of petechial hemorrhages were described, including risk for disseminated intravascular coagulopathy, intramural vascular infiltrates, or SARS-CoV-2 associated endothelitis [15]. Necrotizing encephalopathy is not an uncommon cause for neurological symptoms in COVID-19. Typically affecting the cerebral cortex, the involvement of thalamus, brainstem, and the cerebellum is also reported. Management includes treatment of intravenous immunoglobulins and high-dose corticosteroids [16, 17]. Autoimmune encephalitis and cerebellitis were also reported in those with COVID-19 infection $[18,19]$.

Autoimmune encephalitis and cerebellitis were also reported in those with COVID-19 infection [18, 19]. One systematic review examined the association of myoclonus and cerebellar ataxia (CA) with COVID-19 from 51 patients [21]. The mean age was 59.6 years, ranging from 26 to 88 years, and $21.6 \%$ were female. The median latency between COVID-19 symptoms and myoclonus/ CAs was 13 days, usually within 1 month of COVID-19 symptoms. Among these 51 patients, 23.5\% (12/51) of cases had myoclonus and CAs, 35.3\% (18/51) of cases had myoclonus without CAs, and $41.2 \%$ (21/51) of cases had CAs. Myoclonus and CAs had an acute onset. Myoclonus was multifocal or generalized, activated by action in $56.7 \%$ $(17 / 30)$ of cases and by sensory stimuli in $46.7 \%(14 / 30)$ of cases. Myoclonus and CAs were concurrently associated with other neurological symptoms, including cognitive changes $(45.5 \%)$ or a Miller Fisher syndrome variant (21.2\%). MRI studies were generally unremarkable. Myoclonus and CAs are attributed not to the direct invasion of SARS-CoV-2, but immune-mediated mechanisms, due to the absence of SARS-CoV-2 RNA or intrathecal SARSCoV-2-specific antibody production in most of the patients [20], the association of anti-Purkinje cell antibody [21], and the benefits of immunotherapies [20,21]. Notably, the systematic review reported that $80 \%$ (24/30) of cases showed improvement or resolution of these neurological symptoms within 2 months, either spontaneously or with anti-epileptic therapies and immunotherapies including methylprednisolone and IVIg [16, 17, 21], suggesting immune-mediated functional disorders. It remains unclear the relevance of the COVID-19-associated myoclonus with the perivascular lymphocytic inflammation.

The "long COVID" characterizes persistent or recurrent functional deficits after the recovery from the acute phase of COVID-19 infection. The mechanistic basis of long COVID is characterized by the hypometabolic pattern on [18F]-FDG brain PET imaging. The hypometabolic pattern is a cortical signature and is seen in the brainstem and the cerebellum [22]. Bilateral hypometabolism is common in bilateral rectal/orbital gyrus, the olfactory gyrus, and the right temporal lobe, including the amygdala and the hippocampus [23]. It is also seen in the thalamus, pons/medulla brainstem, and cerebellum. The metabolic clusters of the hypometabolic pattern 
are highly suggestive of long COVID state compared to healthy ones. Furthermore, the hypometabolism is associated with the patients' symptoms, but they do not carry any value as an objective biomarker [23].

Post-COVID cerebellar ataxia was described in one patient who had appendicular and trunk ataxia along with dysarthria 1 day after the resolution of respiratory symptoms [24]. MRI revealed mild cerebellar atrophy. Although post-COVID immune hyperreactivity as a cause of such functional and anatomical deficits cannot be excluded, it is possible that subclinical cerebellar degenerative disease preexisted in the patient who had COVID-19-induced decompensation. Recovery of respiratory symptoms after COVID-19 can be associated with the central nervous system manifestation of the hypercoagulable state, particularly the stroke. In one example, florid neuropsychiatric syndrome was found, including paranoia, irritability, aggression, and disinhibition. The deficit required treatment with antipsychotics and the patient was transferred to neurorehabilitation [25].

COVID-19 had negatively impacted the ongoing care of those with preexisting chronic cerebellar disorders. The number of cerebellar ataxia patients on chronic immunemodulatory therapy was thought to be at high risk of developing severe complications. The immunomodulatory treatment of these patients was interrupted, modified, or delayed. COVID-19 consistently influenced ongoing clinical care and rehabilitation, particularly due to lack of transportation, physical distancing, and social isolation [3, 4]. A structured electronic survey interviewing patients with Friedreich ataxia revealed that interruption in physical therapy led to substantial worsening in $60 \%$ of the patients [26]. Home-based strategies were offered using technology-based tools, but it was only adopted by $30 \%$ of mildly affected patients [26]. The physical and mental health of patients with chronic cerebellar disorder was also at greater risk due to prevailing uncertainty, anxiety, and depression due to lack of social interaction during the lockdown. Online questionnaires surveying SCA patients for self-rating of anxiety, depression, and overall baseline morbidity due to their ataxia revealed a significant increase in anxiety to $34.9 \%$ and depression to $56.7 \%$ [27].

To summarize, COVID-19 significantly affects the human nervous system; the cerebellum is not spared, as initially believed. The effects of COVID-19 on the cerebellum are direct via hematological, vascular, immune, and metabolic mechanisms. The indirect effects of the pandemic were also prominent, as it had deprived the patients with chronic cerebellar ataxias of in-person clinical and neurological care. The novel interventions were helpful but only in selected patients with cerebellar ataxias.

\section{References}

1. Huang C, Wang Y, Li X, Ren L, Zhao J, Hu Y, Zhang L, Fan G, Xu J, Gu X, Cheng Z, Yu T, Xia J, Wei Y, Wu W, Xie X, Yin W, Li H, Liu M, Xiao Y, Gao H, Guo L, Xie J, Wang G, Jiang R, Gao Z, Jin Q, Wang J, Cao B. Clinical features of patients infected with 2019 novel coronavirus in Wuhan. China Lancet. 2020;395:497506. https://doi.org/10.1016/S0140-6736(20)30183-5.

2. Mao L, Jin H, Wang M, Hu Y, Chen S, He Q, Chang J, Hong C, Zhou Y, Wang D, Miao X, Li Y, Hu B. Neurologic manifestations of hospitalized patients with coronavirus disease 2019 in Wuhan. China JAMA Neurol. 2020;77:683-90. https://doi.org/10.1001/ jamaneurol.2020.1127.

3. Manto M, Dupre N, Hadjivassiliou M, Louis ED, Mitoma H, Molinari M, Shaikh AG, Soong BW, Strupp M, Van Overwalle F, Schmahmann JD. Medical and paramedical care of patients with cerebellar ataxia during the COVID-19 outbreak: seven practical recommendations of the COVID 19 Cerebellum Task Force. Front Neurol. 2020;11:516. https://doi.org/10.3389/fneur.2020.00516.

4. Manto M, Dupre N, Hadjivassiliou M, Louis ED, Mitoma H, Molinari M, Shaikh AG, Soong BW, Strupp M, Van Overwalle F, Schmahmann JD. Management of patients with cerebellar ataxia during the COVID-19 pandemic: current concerns and future implications. Cerebellum. 2020;19:562-8. https://doi.org/10. 1007/s12311-020-01139-1.

5. Shaikh AG, Bronstein A, Carmona S, Cha YH, Cho C, Ghasia FF, Gold D, Green KE, Helmchen C, Ibitoye RT, Kattah J, Kim JS, Kothari S, Manto M, Seemungal BM, Straumann D, Strupp M, Szmulewicz D, Tarnutzer A, Tehrani A, Tilikete C, Welgampola M, Zalazar G, Kheradmand A. Consensus on virtual management of vestibular disorders: urgent versus expedited care. Cerebellum. 2021;20:4-8. https://doi.org/10.1007/s12311-020-01178-8.

6. Shaikh AG, Mitoma H, Manto M. Cerebellar scholars' challenging time in COVID-19 pandemia. Cerebellum. 2020;19:343-4. https://doi.org/10.1007/s12311-020-01131-9.

7. Liotta EM, Batra A, Clark JR, Shlobin NA, Hoffman SC, Orban ZS, Koralnik IJ. Frequent neurologic manifestations and encephalopathy-associated morbidity in COVID-19 patients. Ann Clin Transl Neurol. 2020;7:2221-30. https://doi.org/10.1002/acn3. 51210.

8. Li YC, Zhang Y, Tan BH. What can cerebrospinal fluid testing and brain autopsies tell us about viral neuroinvasion of SARSCoV-2. J Med Virol. 2021;93:4247-57. https://doi.org/10.1002/ jmv.26943.

9. Siddiqui R, Mungroo MR, Khan NA. SARS-CoV-2 invasion of the central nervous: a brief review. Hosp Pract. 1995;2021(49):15763. https://doi.org/10.1080/21548331.2021.1887677.

10. Matschke J, Lutgehetmann M, Hagel C, Sperhake JP, Schroder AS, Edler C, Mushumba H, Fitzek A, Allweiss L, Dandri M, Dottermusch M, Heinemann A, Pfefferle S, Schwabenland M, Sumner Magruder D, Bonn S, Prinz M, Gerloff C, Puschel K, Krasemann S, Aepfelbacher M, Glatzel M. Neuropathology of patients with COVID-19 in Germany: a post-mortem case series. Lancet Neurol. 2020;19:919-29. https://doi.org/10.1016/S1474-4422(20) 30308-2.

11. Colombo D, Falasca L, Marchioni L, Tammaro A, Adebanjo GAR, Ippolito G, Zumla A, Piacentini M, Nardacci R, Del Nonno F. Neuropathology and inflammatory cell characterization in 10 autoptic COVID-19 brains. Cells. 2021;10(9):2262. https://doi. org/10.3390/cells10092262.

12. Malik P, Patel K, Akrmah M, Donthi D, Patel U, Khader SN, Asiry S. COVID-19: a disease with a potpourri of histopathologic findings-a literature review and comparison to the closely related SARS and MERS. SN Compr Clin Med 2021;1-28. https://doi. org/10.1007/s42399-021-01029-5. 
13. Meo SA, Abukhalaf AA, Alomar AA, Al-Hussain F. Magnetic resonance imaging (MRI) and neurological manifestations in SARSCoV-2 patients. Eur Rev Med Pharmacol Sci. 2021;25:1101-8. https://doi.org/10.26355/eurrev_202101_24681.

14. Parsons N, Outsikas A, Parish A, Clohesy R, D'Aprano F, Toomey F, Advani S, Poudel GR. Modelling the anatomic distribution of neurologic events in patients with COVID-19: a systematic review of MRI findings. AJNR Am J Neuroradiol. 2021;42:1190-5. https://doi.org/10.3174/ajnr.A7113.

15. Kirschenbaum D, Imbach LL, Rushing EJ, Frauenknecht KBM, Gascho D, Ineichen BV, Keller E, Kohler S, Lichtblau M, Reimann RR, Schreib K, Ulrich S, Steiger P, Aguzzi A, Frontzek $\mathrm{K}$. Intracerebral endotheliitis and microbleeds are neuropathological features of COVID-19. Neuropathol Appl Neurobiol. 2021;47:454-9. https://doi.org/10.1111/nan.12677.

16. Ciolac D, Crivorucica I, Zota E, Gorincioi N, Efremova D, Manea D, Crivorucica V, Ciocanu M, Groppa SA. Extensive cerebellar involvement and cognitive impairment in COVID-19-associated acute necrotizing encephalopathy. Ther Adv Neurol Disord. 2021;14:1756286420985175. https://doi.org/10.1177/1756286420 985175.

17. Shah P, Patel J, Soror NN, Kartan R. Encephalopathy in COVID19 patients. Cureus. 2021;13:e16620. https://doi.org/10.7759/ cureus. 16620 .

18. Fadakar N, Ghaemmaghami S, Masoompour SM, Shirazi Yeganeh B, Akbari A, Hooshmandi S, Ostovan VR. A first case of acute cerebellitis associated with coronavirus disease (COVID-19): a case report and literature review. Cerebellum. 2020;19:911-4. https://doi.org/10.1007/s12311-020-01177-9.

19. Grimaldi S, Lagarde S, Harle JR, Boucraut J, Guedj E. Autoimmune encephalitis concomitant with SARS-CoV-2 infection: insight from (18)F-FDG PET imaging and neuronal autoantibodies. J Nucl Med. 2020;61:1726-9. https://doi.org/10.2967/jnumed. 120.249292

20. Sanchez CV, Theel E, Binnicker M, Toledano M, McKeon A. Autoimmune encephalitis post-SARS-CoV-2 infection: case frequency, findings, and outcomes. Neurology. 2021. https://doi.org/ 10.1212/WNL.0000000000012931.
21. Chan JL, Murphy KA, Sarna JR. Myoclonus and cerebellar ataxia associated with COVID-19: a case report and systematic review. J Neurol. 2021;268:3517-48. https://doi.org/10.1007/ s00415-021-10458-0.

22. Morand A, Campion JY, Lepine A, Bosdure E, Luciani L, Cammilleri S, Chabrol B, Guedj E. Similar patterns of [(18)F]-FDG brain PET hypometabolism in paediatric and adult patients with long COVID: a paediatric case series. Eur J Nucl Med Mol Imaging. 2021. https://doi.org/10.1007/s00259-021-05528-4.

23. Guedj E, Campion JY, Dudouet P, Kaphan E, Bregeon F, Tissot-Dupont H, Guis S, Barthelemy F, Habert P, Ceccaldi M, Million M, Raoult D, Cammilleri S, Eldin C. (18)F-FDG brain PET hypometabolism in patients with long COVID. Eur J Nucl Med Mol Imaging. 2021;48:2823-33. https://doi.org/10.1007/ s00259-021-05215-4.

24. Werner J, Reichen I, Huber M, Abela IA, Weller M, Jelcic I. Subacute cerebellar ataxia following respiratory symptoms of COVID19: a case report. BMC Infect Dis. 2021;21:298. https://doi.org/ 10.1186/s12879-021-05987-y.

25. Chia KX, Polakhare S, Bruno SD. Possible affective cognitive cerebellar syndrome in a young patient with COVID-19 CNS vasculopathy and stroke. BMJ Case Rep. 2020;13(10):e237926. http s://doi.org/10.1136/bcr-2020-237926.

26. Schirinzi T, Sancesario A, Castelli E, Bertini E, Vasco G. Friedreich ataxia in COVID-19 time: current impact and future possibilities. Cerebellum Ataxias. 2021;8:4. https://doi.org/10.1186/ s40673-020-00127-9.

27. Gong Y, Chen Z, Liu M, Wan L, Wang C, Peng H, Shi Y, Peng Y, Xia K, Qiu R, Tang B, Jiang H. Anxiety and depression in spinocerebellar ataxia patients during the COVID-19 pandemic in China: a cross-sectional study. J Clin Neurosci. 2021;88:39-46. https://doi.org/10.1016/j.jocn.2021.03.004.

Publisher's Note Springer Nature remains neutral with regard to jurisdictional claims in published maps and institutional affiliations. 